\title{
MULTIPLE SOLUTIONS FOR A $P(X)$-LAPLACIAN-LIKE PROBLEM WITH NEUMANN CONDITION ORIGINATED FROM A CAPILLARY PHENOMENA
}

\author{
S. Heidarkhani, A.L.A. De Araujo, G.A. Afrouzi, S. Moradi
}

ABstRaCT. This paper presents several sufficient conditions for the existence of at least three weak solutions for the following Neumann problem, originated from a capillary phenomena,

$$
\begin{cases}-\operatorname{div}\left(\left(1+\frac{|\nabla u|^{p(x)}}{\sqrt{1+|\nabla u|^{2 p(x)}}}\right)|\nabla u|^{p(x)-2} \nabla u\right)+a(x)|u|^{p(x)-2} u & \\ =\lambda f(x, u)+\mu g(x, u), & \text { in } \Omega, \\ \frac{\partial u}{\partial \nu}=0, & \text { on } \partial \Omega\end{cases}
$$

where $\Omega \subset \mathbb{R}^{N}(N \geq 2)$ is a bounded domain with boundary of class $C^{1}, \nu$ is the outer unit normal to $\partial \Omega, \lambda>0, \mu \geq 0, a \in L^{\infty}(\Omega), f, g: \Omega \times \mathbb{R} \rightarrow \mathbb{R}$ are two $L^{1}$-Carathéodory functions and $p \in C^{0}(\bar{\Omega})$. Our technical approach is based on variational methods. The main result is also demonstrated with an example.

2010 Mathematics Subject Classification: 35D30, 35J60.

Keywords: Variable exponent spaces, $p(x)$-Laplacian-like, Three solutions, Variational methods.

\section{INTRODUCTION}

In this paper, we are interested in establishing the existence of at least three weak solutions to the following $p(x)$-Laplacian-like problem, originated from a capillary phenomena,

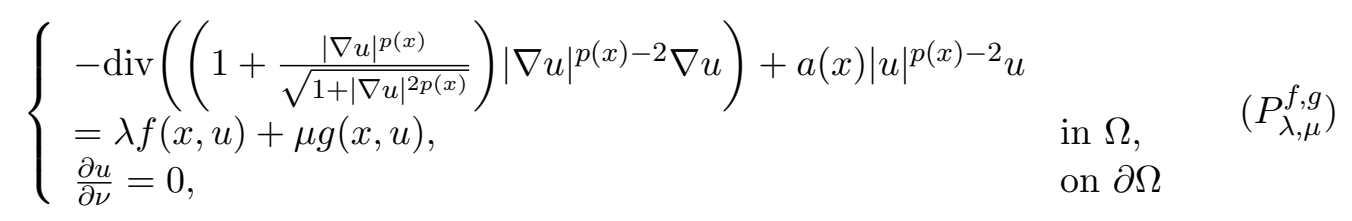


where $\Omega \subset \mathbb{R}^{N}(N \geq 2)$ is a bounded domain with boundary of class $C^{1}, \nu$ is the outer unit normal to $\partial \Omega, \lambda>0, \mu \geq 0, a \in L^{\infty}(\Omega)$ with $\operatorname{essinf}_{\Omega} a \geq 0$, $f, g: \Omega \times \mathbb{R} \rightarrow \mathbb{R}$ are two $L^{1}$-Carathéodory functions and $p \in C^{0}(\bar{\Omega})$ satisfies the condition

$$
N<p^{-}:=\inf _{x \in \bar{\Omega}} p(x) \leq p^{+}:=\sup _{x \in \bar{\Omega}} p(x)<+\infty .
$$

During the last fifteen years, differential and partial differential equations with variable exponent growth conditions have become increasingly popular. This is partly due to their frequent appearance in applications such as the modeling of electrorheological fluids [41], image restoration [13], elastic mechanics [45] and continuum mechanics [2], but these problems are very interesting from a purely mathematical point of view as well. For a general account of the underlying physics see [23] and for some technical applications [37]. Electrorheological fluids also have functions in robotics and space technology. For background and recent results, we refer the reader to $[11,15,17,18,34,38,39,40]$ and the references therein for details. For example, Fan and Deng in [18] studied the existence and multiplicity of positive solutions for the inhomogeneous Neumann boundary value problems involving the $p(x)$-Laplacian of the following form

$$
\begin{cases}-\operatorname{div}\left(|\nabla u|^{p(x)-2} \nabla u\right)+\lambda|u|^{p(x)-2} u=f(x, u), & \text { in } \Omega, \\ |\nabla u|^{p(x)-2} \frac{\partial u}{\partial \eta}=\varphi, & \text { on } \partial \Omega\end{cases}
$$

where $\Omega$ is a bounded smooth domain in $\mathbb{R}^{N}, p \in C^{1}(\Omega)$ and $p(x)>1$ for $x \in \Omega$, $\varphi \in C^{0, \gamma}(\partial \Omega)$ with $\gamma \in(0,1), \varphi \geq 0$ and $\varphi \neq 0$ on $\partial \Omega$, they by using the subsupersolution method and the variational method, under appropriate assumptions on $f$, obtained there exists $\lambda_{*}>0$ such that the problem has at least two positive solutions if $\lambda>\lambda_{*}$, has at least one positive solution if $\lambda=\lambda_{*}$, and has no positive solution if $\lambda<\lambda_{*}$. Deng in [17] by using a local mountain pass theorem without (P.S) condition and Ricceri's variational principle, obtained the existence and multiplicity of non-trivial solutions for the following $p(x)$-Laplacian double perturbed Neumann problem with nonlinear boundary condition

$$
\begin{cases}-\Delta_{p(x)} u+a(x)|u|^{p(x)-2} u=f(x, u)+\lambda h_{1}(x, u), & \text { in } \Omega, \\ |\nabla u|^{p(x)-2} \frac{\partial u}{\partial \gamma}=g(x, u)+\mu h_{2}(x, u), & \text { on } \partial \Omega\end{cases}
$$

where $\Omega$ is a bounded open domain in $\mathbb{R}^{N}$ with smooth boundary, $-\Delta_{p(x)} u=$ $-\operatorname{div}\left(|\nabla u|^{p(x)-2} \nabla u\right)$ is the $p(x)$-Laplacian with $p \in C(\bar{\Omega}), p(x)>1, \lambda, \mu \in \mathbb{R}$, $a \in L^{\infty}(\Omega)$ with ess $\inf _{x \in \Omega} a(x)=a^{-}>0, \gamma$ is the outward unit normal to $\partial \Omega$. Cammaroto and Vilasi in [11] by using as main tool, a recent variational principle due to Ricceri, established the existence of at least three solutions for the Neumann 
S. Heidarkhani, A.L.A. De Araujo, G.A. Afrouzi, S. Moradi - Multiple ...

problem involving the $p(x)$-Laplacian operator. D'Aguì and Sciammetta in [15] based on variational methods established the existence of an unbounded sequence of weak solutions for a class of differential equations with $p(x)$-Laplacian and subject to small perturbations of nonhomogeneous Neumann conditions.

In recent years, existence, non-existence and multiplicity of positive solutions for one dimensional mean curvature problems have been widely investigated. We refer the reader to the papers $[30,31,32,36]$ and the references therein. We just observe that the one-dimensional problem has been rather thoroughly discussed, by using different methods, in some recent papers by Bonanno et al., in [8], Bonheure et al., in [9], Habets and Omari [24], Boreanu and Mawhin in [4], Faraci in [22] and Pan in [35].

Capillarity can be briefly explained by considering the effects of two opposing forces: adhesion, i.e., the attractive (or repulsive) force between the molecules of the liquid and those of the container; and cohesion, i.e., the attractive force between the molecules of the liquid. Recently, the study of capillarity phenomena has been an interesting topic. We refer the reader to $[3,5,10,14,16,25,27,29,33,46]$ for an overview of and references on this subject. For example, Manuela Rodrigues in [29] by using Mountain Pass lemma (see [12]) and Fountain theorem (see Theorem 3.6 in [43]), established the existence of non-trivial solutions for problem

$$
\begin{cases}-\operatorname{div}\left(\left(1+\frac{|\nabla u|^{p(x)}}{\sqrt{1+|\nabla u|^{2 p(x)}}}\right)|\nabla u|^{p(x)-2} \nabla u\right)=\lambda f(x, u), & x \in \Omega, \\ u=0, & x \in \partial \Omega\end{cases}
$$

where $\Omega \subset \mathbb{R}^{N}(N \geq 2)$ is a bounded domain with boundary of class $C^{1}, \lambda$ is a positive parameter, $p \in C(\bar{\Omega})$ and $f$ is a Carathéodory function. Bin in [5] obtained the existence results of non-trivial solutions for every parameter $\lambda$ for the nonlinear eigenvalue problems for $p(x)$-Laplacian-like operators originated from a capillary phenomena of the following form:

$$
\begin{cases}-\operatorname{div}\left(\left(1+\frac{|\nabla u|^{p(x)}}{\sqrt{1+|\nabla u|^{2 p(x)}}}\right)|\nabla u|^{p(x)-2} \nabla u\right)=\lambda f(x, u), & \text { in } \Omega, \\ u=0, & \text { on } \partial \Omega\end{cases}
$$

where $\Omega \subset \mathbb{R}^{N}$ is a bounded domain with smooth boundary $\partial \Omega, \lambda>0$ is a parameter. Firstly, by using the mountain pass theorem a nontrivial solution is constructed for almost every parameter $\lambda>0$. Then, he considered the continuation of the solutions. Zhou in [46] by employing variational methods, established the existence of at least one non-trivial solution for the following nonlinear eigenvalue problem for the $p(x)$ - 
Laplacian-like operators originated from a capillary phenomenon

$$
\begin{cases}-\operatorname{div}\left(\left(1+\frac{|\nabla u|^{p(x)}}{\sqrt{1+|\nabla u|^{2 p(x)}}}\right)|\nabla u|^{p(x)-2} \nabla u\right)=\lambda f(x, u), & \text { in } \Omega, \\ u=0, & \text { on } \partial \Omega\end{cases}
$$

where $\Omega$ is a bounded domain in $\mathbb{R}^{N}$ with smooth boundary $\partial \Omega, p \in C(\bar{\Omega}), \lambda>0$ is a parameter, $f \in C(\bar{\Omega} \times \mathbb{R})$ is superlinear and do not satisfy the Ambrosetti and Rabinowitz type condition. The existence of at least one nontrivial solution was also proved. In [25] by using variational methods, the existence of at least one weak solution and infinitely many weak solutions for the problem $\left(P_{\lambda, \mu}^{f, g}\right)$, in case $\mu=0$ was discussed. In [27] the multiplicity results for the problem $\left(P_{\lambda, \mu}^{f, g}\right)$, in case $\mu=0$ were established. In fact, using a consequence of the local minimum theorem due Bonanno and mountain pass theorem the existence results for the problem under algebraic conditions with the classical Ambrosetti-Rabinowitz (AR) condition on the nonlinear term were obtained. Furthermore, by combining two algebraic conditions on the nonlinear term employing two consequences of the local minimum theorem due Bonanno the existence of two solutions was guaranteed, applying the mountain pass theorem given by Pucci and Serrin the existence of third solution for the problem $\left(P_{\lambda, \mu}^{f, g}\right)$, in case $\mu=0$ was discussed.

Inspired by the above results, in the present paper, we obtain the existence of at least three weak solutions for the problem $\left(P_{\lambda, \mu}^{f, g}\right)$, in which two parameters are involved. Precise estimates of these two parameters $\lambda$ and $\mu$ will be given. In Theorem 3 we establish the existence of at least three weak solutions for the problem $\left(P_{\lambda, \mu}^{f, g}\right)$. In particular, we require that there is a growth of the antiderivative of $f$ which is greater than quadratic in a suitable interval (see, for instance, condition $\left(\mathrm{A}_{4}\right)$ of Theorem 4$)$, and which is less than quadratic in a following suitable interval (see, for instance, condition $\left(\mathrm{A}_{4}\right)$ of Theorem 4). We present Example 1 in which the hypotheses of Theorem 4 are fulfilled. Theorem 5 is a simple consequence of Theorem 4. As a special case of Theorem 4, we obtain Theorem 6 in the case $f$ does not depend upon $x$. Theorems 7 and 8 , under suitable conditions on $f$ at zero and at infinity, ensure four distinct non-trivial solutions to the problem $\left(P_{\lambda, \mu}^{f, g}\right)$ when $\mu=0$. As a special case of Theorem 3, we obtain Theorem 9 considering the case $p(x)=p>N$.

The remainder of the paper is organized as follows. In Section 2, we will recall the definitions and some properties of variable exponent Sobolev spaces. In Section 3 we will state and prove the main results of the paper. 
S. Heidarkhani, A.L.A. De Araujo, G.A. Afrouzi, S. Moradi - Multiple ...

\section{Preliminaries}

Let $X$ be a nonempty set and $\Phi, \Psi: X \rightarrow \mathbb{R}$ be two functions. For all $r, r_{1}, r_{2}>$ $\inf _{X} \Phi, r_{2}>r_{1}, r_{3}>0$, we define

$$
\begin{gathered}
\varphi(r):=\inf _{u \in \Phi^{-1}(]-\infty, r[)} \frac{\left(\sup _{u \in \Phi^{-1}(]-\infty, r[)} \Psi(u)\right)-\Psi(u)}{r-\Phi(u)}, \\
\beta\left(r_{1}, r_{2}\right):=\inf _{u \in \Phi^{-1}(]-\infty, r_{1}[)} \sup _{v \in \Phi^{-1}\left(\left[r_{1}, r_{2}[)\right.\right.} \frac{\Psi(v)-\Psi(u)}{\Phi(v)-\Phi(u)}, \\
\gamma\left(r_{2}, r_{3}\right):=\frac{\sup _{u \in \Phi^{-1}(]-\infty, r_{2}+r_{3}[)} \Psi(u)}{r_{3}}, \\
\alpha\left(r_{1}, r_{2}, r_{3}\right):=\max \left\{\varphi\left(r_{1}\right), \varphi\left(r_{2}\right), \gamma\left(r_{2}, r_{3}\right)\right\} .
\end{gathered}
$$

Theorem 1 ([6], Theorem 3.3). Let $X$ be a reflexive real Banach space, $\Phi: X \rightarrow$ $\mathbb{R}$ be a convex, coercive and continuously Gâteaux differentiable functional whose Gâteaux derivative admits a continuous inverse on $X^{*}, \Psi: X \rightarrow \mathbb{R}$ be a continuously Gâteaux differentiable functional whose Gâteaux derivative is compact, such that

$\left(a_{1}\right) \inf _{X} \Phi=\Phi(0)=\Psi(0)=0$

$\left(a_{2}\right)$ for every $u_{1}, u_{2} \in X$ such that $\Psi\left(u_{1}\right) \geq 0$ and $\Psi\left(u_{2}\right) \geq 0$, one has

$$
\inf _{s \in[0,1]} \Psi\left(s u_{1}+(1-s) u_{2}\right) \geq 0 .
$$

Assume that there are three positive constants $r_{1}, r_{2}, r_{3}$ with $r_{1}<r_{2}$, such that

$\left(a_{3}\right) \varphi\left(r_{1}\right)<\beta\left(r_{1}, r_{2}\right)$

$\left(a_{4}\right) \varphi\left(r_{2}\right)<\beta\left(r_{1}, r_{2}\right)$

$\left(a_{5}\right) \gamma\left(r_{2}, r_{3}\right)<\beta\left(r_{1}, r_{2}\right)$.

Then, for each $\lambda \in] \frac{1}{\beta\left(r_{1}, r_{2}\right)}, \frac{1}{\alpha\left(r_{1}, r_{2}, r_{3}\right)}[$ the functional $\Phi-\lambda \Psi$ admits three distinct critical points $u_{1}, u_{2}, u_{3}$ such that $u_{1} \in \Phi^{-1}(]-\infty, r_{1}[), u_{2} \in \Phi^{-1}\left(\left[r_{1}, r_{2}[)\right.\right.$ and $u_{3} \in \Phi^{-1}(]-\infty, r_{2}+r_{3}[)$.

We refer the interested reader to the papers $[1,7,26]$ in which Theorem 1 has been successfully employed to obtain the existence of at least three solutions for boundary value problems.

For the reader's convenience, we state some basic properties of variable exponent Sobolev spaces and introduce some notations. For other basic notations 
S. Heidarkhani, A.L.A. De Araujo, G.A. Afrouzi, S. Moradi - Multiple ...

and definitions, and for a thorough account on the subject, we refer the reader to $[19,20,21,28,41,42]$.

Set

$$
C_{+}(\Omega):=\{h \in C(\bar{\Omega}): h(x)>1, \forall x \in \bar{\Omega}\} .
$$

For $p \in C_{+}(\Omega)$, define

$$
L^{p(x)}(\Omega):=\left\{u: \Omega \rightarrow \mathbb{R} \text { measurable and } \int_{\Omega}|u(x)|^{p(x)} d x<+\infty\right\} .
$$

We can introduce a norm on $L^{p(x)}(\Omega)$ by

$$
|u|_{p(x)}=\inf \left\{\beta>0: \int_{\Omega}\left|\frac{u(x)}{\beta}\right|^{p(x)} d x \leq 1\right\} .
$$

The space $\left(L^{p(x)}(\Omega),|u|_{p(x)}\right)$ is a Banach space called a variable exponent Lebesgue space. Define the Sobolev space with variable exponent

$$
W^{1, p(x)}(\Omega)=\left\{u \in L^{p(x)}(\Omega):|\nabla u| \in L^{p(x)}(\Omega)\right\}
$$

equipped with the norm

$$
\|u\|_{1, p(x)}:=|u|_{p(x)}+|\nabla u|_{p(x)} .
$$

$W^{1, p(x)}(\Omega)$ is a separable and reflexive Banach space (see [20]).

When $a \in L^{\infty}(\Omega)$ with $\operatorname{ess} \inf _{\Omega} a \geq 0$, we define

$$
L_{a(x)}^{p(x)}(\Omega):=\left\{u: \Omega \rightarrow \mathbb{R} \text { measurable and } \int_{\Omega} a(x)|u(x)|^{p(x)} d x<+\infty\right\}
$$

with the norm

$$
|u|_{p(x), a(x)}=\inf \left\{\beta>0: \int_{\Omega} a(x)\left|\frac{u(x)}{\beta}\right|^{p(x)} d x \leq 1\right\}
$$

For any $u \in W^{1, p(x)}(\Omega)$, define

$$
\|u\|_{a}:=\inf \left\{\beta>0: \int_{\Omega}\left(\left|\frac{\nabla u(x)}{\beta}\right|^{p(x)}+a(x)\left|\frac{u(x)}{\beta}\right|^{p(x)}\right) d x \leq 1\right\} .
$$

Then, it is easy to see that $\|u\|_{a}$ is a norm on $W^{1, p(x)}(\Omega)$ equivalent to $\|u\|_{1, p(x)}$. In the following, we will use $\|\cdot\|_{a}$ instead of $\|\cdot\|_{1, p(x)}$ on $X=W^{1, p(x)}(\Omega)$. 
S. Heidarkhani, A.L.A. De Araujo, G.A. Afrouzi, S. Moradi - Multiple ...

As pointed out in [21] and [28], $X$ is continuously embedded in $W^{1, p^{-}}(\Omega)$ and, since $p^{-}>N, W^{1, p^{-}}(\Omega)$ is compactly embedded in $C^{0}(\bar{\Omega})$. Thus, $X$ is compactly embedded in $C^{0}(\bar{\Omega})$. So, in particular, there exists a positive constant $k>0$ such that

$$
\|u\|_{C^{0}(\bar{\Omega})} \leq k\|u\|_{a}
$$

for each $u \in X$. When $\Omega$ is convex, an explicit upper bound for the constant $k$ is

$$
k \leq 2^{\frac{p^{-}-1}{p^{-}}} \max \left\{\left(\frac{1}{\|a\|_{1}}\right)^{\frac{1}{p^{-}}}, \frac{\sigma}{N^{\frac{1}{p^{-}}}}\left(\frac{p^{-}-1}{p^{-}-N} \operatorname{meas}(\Omega)\right)^{\frac{p^{-}-1}{p^{-}}} \frac{\|a\|_{\infty}}{\|a\|_{1}}\right\}(1+\operatorname{meas}(\Omega)),
$$

where $\sigma=\operatorname{diam}(\Omega)$ and meas $(\Omega)$ is the Lebesgue measure of $\Omega$ (for details, see [15]), $\|a\|_{1}=\int_{\Omega} a(x) d x$ and $\|a\|_{\infty}=\sup _{x \in \Omega} a(x)$.

Lemma $2([21])$. Set $\rho(u)=\int_{\Omega}\left(|\nabla u(x)|^{p(x)}+a(x)|u(x)|^{p(x)}\right) d x$. For $u \in X$ we have

(i) $\|u\|_{a}<(=;>) 1 \Leftrightarrow \rho(u)<(=;>) 1$

(ii) $\|u\|_{a}<1 \Rightarrow\|u\|_{a}^{p^{+}} \leq \rho(u) \leq\|u\|_{a}^{p^{-}}$,

(iii) $\|u\|_{a}>1 \Rightarrow\|u\|_{a}^{p^{-}} \leq \rho(u) \leq\|u\|_{a}^{p^{+}}$.

We need the following proposition in the proofs of our main results.

Proposition 1 ([29]). The functional $\Phi: X \rightarrow \mathbb{R}$ is convex and the mapping $\Phi^{\prime}: X \rightarrow X^{*}$ is a strictly monotone and bounded homeomorphism.

Corresponding to the functions $f$ and $g$, we introduce the functions $F: \Omega \times \mathbb{R} \rightarrow$ $\mathbb{R}$ and $G: \Omega \times \mathbb{R} \rightarrow \mathbb{R}$, respectively, as follows

$$
F(x, t)=\int_{0}^{t} f(x, \xi) d \xi \text { for all }(x, t) \in \Omega \times \mathbb{R}
$$

and

$$
G(x, t)=\int_{0}^{t} g(x, \xi) d \xi \text { for all }(x, t) \in \Omega \times \mathbb{R} .
$$

Moreover, set $G^{\theta}:=\int_{\Omega} \sup _{|t| \leq \theta} G(x, t) d x$ for every $\theta>0$ and $G_{\eta}:=\inf _{\Omega \times[0, \eta]} G(x, t)$ for every $\eta>0$.

If $g$ is sign-changing, then $G^{\theta} \geq 0$ and $G_{\eta} \leq 0$. 
S. Heidarkhani, A.L.A. De Araujo, G.A. Afrouzi, S. Moradi - Multiple ...

\section{MAIN RESUlts}

We present our main result as follows.

We fix four positive constants $\theta_{1} \geq k, \theta_{2}, \theta_{3}$ and $\eta \geq 1$, put

$$
\begin{aligned}
& \delta_{\lambda, g}:=\min \left\{\frac { 1 } { k ^ { p ^ { - } } p ^ { + } } \operatorname { m i n } \left\{\frac{\theta_{1}^{p^{-}}-\lambda k^{p^{-}} p^{+} \int_{\Omega} F\left(x, \theta_{1}\right) d x}{G^{\theta_{1}}}\right.\right. \\
& \left., \frac{\theta_{2}^{p^{-}}-\lambda k^{p^{-}} p^{+} \int_{\Omega} F\left(x, \theta_{2}\right) d x}{G^{\theta_{2}}}, \frac{\left(\theta_{3}^{p^{-}}-\theta_{2}^{p^{-}}\right)-\lambda k^{p^{-}} p^{+} \int_{\Omega} F\left(x, \theta_{3}\right) d x}{G^{\theta_{3}}}\right\} \\
& \left., \frac{\frac{\eta^{p^{+}}}{p^{-}}\|a\|_{1}-\lambda\left(\int_{\Omega} F(x, \eta) d x-\int_{\Omega} F\left(x, \theta_{1}\right) d x\right)}{G_{\eta}-G^{\theta_{1}}}\right\} .
\end{aligned}
$$

Theorem 3. Assume that there exist positive constants $\theta_{1} \geq k, \theta_{2}, \theta_{3}$ and $\eta \geq 1$ with $\theta_{1}<\sqrt[p]{\|a\|_{1}} k \eta$ and $\max \left\{\eta, \sqrt[p]{\frac{p^{+}\|a\|_{1}}{p^{-}}} k \eta^{\frac{p^{+}}{p^{-}}}\right\}<\theta_{2}$ and $\theta_{2}<\theta_{3}$ such that

$\left(A_{1}\right) f(x, t) \geq 0$ for each $(x, t) \in \Omega \times\left[-\theta_{3}, \theta_{3}\right]$;

$$
\begin{aligned}
\max & \left\{\frac{\int_{\Omega} F\left(x, \theta_{1}\right) d x}{\theta_{1}^{p^{-}}}, \frac{\int_{\Omega} F\left(x, \theta_{2}\right) d x}{\theta_{2}^{p^{-}}}, \frac{\int_{\Omega} F\left(x, \theta_{3}\right) d x}{\theta_{3}^{p^{-}}-\theta_{2}^{p^{-}}}\right\} \\
& <\frac{p^{-}}{k^{p^{-}} p^{+}\|a\|_{1}} \frac{\int_{\Omega} F(x, \eta) d x-\int_{\Omega} F\left(x, \theta_{1}\right) d x}{\eta^{p^{+}}} .
\end{aligned}
$$

Then, for every

$$
\begin{gathered}
\lambda \in\left(\frac{\frac{\eta^{p^{+}}}{p^{-}}\|a\|_{1}}{\int_{\Omega} F(x, \eta) d x-\int_{\Omega} F\left(x, \theta_{1}\right) d x}\right. \\
\left., \frac{1}{p^{+} k^{p^{-}}} \min \left\{\frac{\theta_{1}^{p^{-}}}{\int_{\Omega} F\left(x, \theta_{1}\right) d x}, \frac{\theta_{2}^{p^{-}}}{\int_{\Omega} F\left(x, \theta_{2}\right) d x}, \frac{\theta_{3}^{p^{-}}-\theta_{2}^{p^{-}}}{\int_{\Omega} F\left(x, \theta_{3}\right) d x}\right\}\right)
\end{gathered}
$$

for every non-negative $L^{1}$-Carathéodory function $g: \Omega \times \mathbb{R} \rightarrow \mathbb{R}$ there exists $\delta_{\lambda, g}>0$ given by (3) such that, for each $\mu \in\left[0, \delta_{\lambda, g}\right)$, the problem $\left(P_{\lambda, \mu}^{f, g}\right)$ possesses at least three non-negative weak solutions $u_{1}, u_{2}$, and $u_{3}$ such that

$$
\max _{x \in \Omega}\left|u_{1}(x)\right|<\theta_{1}, \max _{x \in \Omega}\left|u_{2}(x)\right|<\theta_{2} \text { and } \max _{x \in \Omega}\left|u_{3}(x)\right|<\theta_{3} .
$$


Proof. Our aim is to apply Theorem 1 to our problem. We consider the auxiliary problem

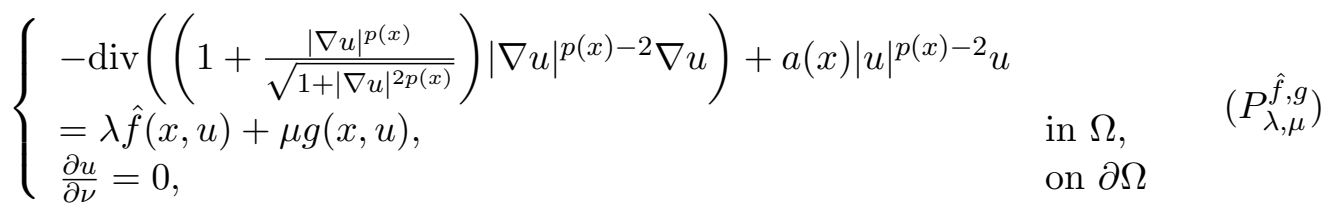

where $\hat{f}: \Omega \times \mathbb{R} \rightarrow \mathbb{R}$ is an $L^{1}$-Carathéodory function, defined as follows

$$
\hat{f}(x, \xi)= \begin{cases}f(x, 0), & \text { if } \xi<-\theta_{3}, \\ f(x, \xi), & \text { if }-\theta_{3} \leq \xi \leq \theta_{3}, \\ f\left(x, \theta_{3}\right), & \text { if } \xi>\theta_{3}\end{cases}
$$

If any solution of the problem $\left(P_{\lambda, \mu}^{f, g}\right)$ satisfies the condition $-\theta_{3} \leq u(x) \leq \theta_{3}$ for every $x \in \Omega$, then, any weak solution of the problem $\left(P_{\lambda, \mu}^{\hat{f}, g}\right)$ clearly turns to be also a weak solution of $\left(P_{\lambda, \mu}^{f, g}\right)$. Therefore, for our goal, it is enough to show that our conclusion holds for $\left(P_{\lambda, \mu}^{f, g}\right)$. Fix $\lambda, g$ and $\mu$ as in the conclusion. In order to apply Theorem 1 to our problem. $\Phi, \Psi$ for every $u \in X$, defined by

$$
\Phi(u):=\int_{\Omega} \frac{1}{p(x)}\left(|\nabla u(x)|^{p(x)}+\sqrt{1+|\nabla u(x)|^{2 p(x)}}+a(x)|u(x)|^{p(x)}\right) d x
$$

and

$$
\Psi(u):=\int_{\Omega} F(x, u(x)) d x+\frac{\mu}{\lambda} \int_{\Omega} G(x, u(x)) d x,
$$

and put $I_{\lambda}(u)=\Phi(u)-\lambda \Psi(u)$ for every $u \in X$. Note that the weak solutions of $\left(P_{\lambda, \mu}^{f, g}\right)$ are exactly the critical points of $I_{\lambda}$. The functionals $\Phi$ and $\Psi$ satisfy the regularity assumptions of Theorem 1. Indeed, similar arguments as in [29] show that $\Phi$ is Gâteaux differentiable and sequentially weakly lower semicontinuous and its Gâteaux derivative is the functional $\Phi^{\prime}(u) \in X^{*}$, given by

$$
\begin{aligned}
\Phi^{\prime}(u)(v) & =\int_{\Omega}\left(|\nabla u(x)|^{p(x)-2} \nabla u(x)+\frac{|\nabla u(x)|^{2 p(x)-2} \nabla u(x)}{\sqrt{1+|\nabla u(x)|^{2 p(x)}}}\right) \nabla v(x) d x \\
& +\int_{\Omega} \alpha(x)|u(x)|^{p(x)-2} u(x) v(x) d x
\end{aligned}
$$

for every $v \in X$. Furthermore, Proposition 1 gives that $\Phi^{\prime}: X \rightarrow X^{*}$ admits a continuous inverse, and Lemma 2 follows that $\Phi$ is coercive. On the other hand, 
S. Heidarkhani, A.L.A. De Araujo, G.A. Afrouzi, S. Moradi - Multiple ...

it is well known that $\Psi$ is a differentiable functional whose differential at the point $u \in X$ is

$$
\Psi^{\prime}(u)(v)=\int_{\Omega}\left(f(x, u(x))+\frac{\mu}{\lambda} g(x, u(x))\right) v(x) d x
$$

for any $v \in X$ as well as it is sequentially weakly upper semicontinuous. Furthermore $\Psi^{\prime}: X \rightarrow X^{*}$ is a compact operator. Indeed, it is enough to show that $\Psi^{\prime}$ is strongly continuous on $X$. For this end, for $u \in X$, let $u_{n} \rightarrow u$ weakly in $X$ as $n \rightarrow \infty$, then $u_{n}$ converges uniformly to $u$ on $\Omega$ as $n \rightarrow \infty$; see [44]. Since $f, g$ are continuous functions in $\mathbb{R}$ for every $x \in \Omega$, so

$$
f\left(x, u_{n}\right)+\frac{\mu}{\lambda} g\left(x, u_{n}\right) \rightarrow f(x, u)+\frac{\mu}{\lambda} g(x, u)
$$

as $n \rightarrow \infty$. Hence $\Psi^{\prime}\left(u_{n}\right) \rightarrow \Psi^{\prime}(u)$ as $n \rightarrow \infty$. Thus we have proved that $\Psi^{\prime}$ is strongly continuous on $X$, which implies that $\Psi^{\prime}$ is a compact operator by Proposition 26.2 of [44]. Put $r_{1}:=\frac{1}{p^{+}}\left(\frac{\theta_{1}}{k}\right)^{p^{-}}, r_{2}:=\frac{1}{p^{+}}\left(\frac{\theta_{2}}{k}\right)^{p^{-}}, r_{3}:=\frac{1}{p^{+}}\left(\frac{\theta_{3}^{p^{-}}-\theta_{2}^{p^{-}}}{k^{p^{-}}}\right)$and $w(x)=\eta$ for all $x \in \Omega$. We clearly observe that $w \in X$. Hence, we have definitively,

$$
\frac{\eta^{p^{-}}}{p^{+}}\|a\|_{1} \leq \Phi(w)=\int_{\Omega} \frac{1}{p(x)}\left(a(x) \eta^{p(x)}\right) d x \leq \frac{\eta^{p^{+}}}{p^{-}}\|a\|_{1} .
$$

From the conditions $\theta_{3}>\theta_{2}, \theta_{1}<\sqrt[p^{-}]{\|a\|_{1}} k \eta$ and $\sqrt[p^{-}]{\frac{p^{+}\|a\|_{1}}{p^{-}}} k \eta^{\frac{p^{+}}{p^{-}}}<\theta_{2}$, we get $r_{3}>0$ and $r_{1}<\Phi(w)<r_{2}$. By Lemma 2 and the fact $\max \left\{r_{1}^{1 / p^{-}}, r_{1}^{1 / p^{+}}\right\}=r_{1}^{1 / p^{-}}$, we deduce

$$
\begin{aligned}
\Phi^{-1}\left(-\infty, r_{1}\right) & =\left\{u \in X: \Phi(u)<r_{1}\right\} \subseteq\left\{u \in X:\|u\|_{a}<r_{1}^{1 / p^{-}}\right\} \\
& =\left\{u \in X:\|u\|_{a}<\frac{\theta_{1}}{k}\right\} .
\end{aligned}
$$

Moreover, due to (2), we have

$$
|u(x)| \leq\|u\|_{\infty} \leq k\|u\|_{a} \leq \theta_{1}, \quad \forall x \in \bar{\Omega} .
$$

Hence,

$$
\left\{u \in X:\|u\|_{a}<\frac{\theta_{1}}{k}\right\} \subseteq\left\{u \in X:\|u\|_{\infty} \leq \theta_{1}\right\}
$$

By using the assumption $\left(A_{1}\right)$, one has

$$
\sup _{u \in \Phi^{-1}\left(-\infty, r_{1}\right)} \int_{\Omega} F(x, u(x)) d x \leq \int_{\Omega} \sup _{|t| \leq \theta_{1}} F(x, t) d x \leq \int_{\Omega} F\left(x, \theta_{1}\right) d x .
$$


S. Heidarkhani, A.L.A. De Araujo, G.A. Afrouzi, S. Moradi - Multiple ...

In a similar way, we have

$$
\sup _{u \in \Phi^{-1}\left(-\infty, r_{2}\right)} \int_{\Omega} F(x, u(x)) d x \leq \int_{\Omega} F\left(x, \theta_{2}\right) d x
$$

and

$$
\sup _{u \in \Phi^{-1}\left(-\infty, r_{2}+r_{3}\right)} \int_{\Omega} F(x, u(x)) d x \leq \int_{\Omega} F\left(x, \theta_{3}\right) d x .
$$

Therefore, since $0 \in \Phi^{-1}\left(-\infty, r_{1}\right)$ and $\Phi(0)=\Psi(0)=0$, one has

$$
\begin{aligned}
\varphi\left(r_{1}\right) & \leq \frac{\sup _{u \in \Phi^{-1}\left(-\infty, r_{1}\right)} \Psi(u)}{r_{1}} \\
& =\frac{\sup _{u \in \Phi^{-1}\left(-\infty, r_{1}\right)}\left[\int_{\Omega} F(x, u(x)) d x+\frac{\mu}{\lambda} \int_{\Omega} G(x, u(x)) d x\right]}{r_{1}} \\
& \leq \frac{\int_{\Omega} F\left(x, \theta_{1}\right) d x+\frac{\mu}{\lambda} G^{\theta_{1}}}{\frac{1}{p^{+}}\left(\frac{\theta_{1}}{k}\right)^{p^{-}}}, \\
\varphi\left(r_{2}\right) & \leq \frac{\sup _{u \in \Phi^{-1}\left(-\infty, r_{2}\right)} \Psi(u)}{r_{2}} \\
& =\frac{\sup _{u \in \Phi^{-1}\left(-\infty, r_{2}\right)}\left[\int_{\Omega} F(x, u(x)) d x+\frac{\mu}{\lambda} \int_{\Omega} G(x, u(x)) d x\right]}{r_{2}} \\
& \leq \frac{\int_{\Omega} F\left(x, \theta_{2}\right) d x+\frac{\mu}{\lambda} G^{\theta_{2}}}{\frac{1}{p^{+}}\left(\frac{\theta_{2}}{k}\right)^{p^{-}}}
\end{aligned}
$$

and

$$
\begin{aligned}
\gamma\left(r_{2}, r_{3}\right) & \leq \frac{\sup _{u \in \Phi^{-1}\left(-\infty, r_{2}+r_{3}\right)} \Psi(u)}{r_{3}} \\
& =\frac{\sup _{u \in \Phi^{-1}\left(-\infty, r_{2}+r_{3}\right)}\left[\int_{\Omega} F(x, u(x)) d x+\frac{\mu}{\lambda} \int_{\Omega} G(x, u(x)) d x\right]}{r_{3}} \\
& \leq \frac{\int_{\Omega} F\left(x, \theta_{3}\right) d x+\frac{\mu}{\lambda} G^{\theta_{3}}}{\frac{1}{p^{+}}\left(\frac{\theta_{3}^{p^{-}}-\theta_{2}^{p^{-}}}{k^{p^{-}}}\right)} .
\end{aligned}
$$

On the other hand, we have

$$
\begin{aligned}
\Psi(w) & =\int_{\Omega} F(x, w(x)) d x+\frac{\mu}{\lambda} \int_{\Omega} G(x, w(x)) d x \\
& \geq \int_{\Omega} F(x, w(x)) d x+\frac{\mu}{\lambda} G_{\eta} .
\end{aligned}
$$


S. Heidarkhani, A.L.A. De Araujo, G.A. Afrouzi, S. Moradi - Multiple ...

For each $u \in \Phi^{-1}\left(-\infty, r_{1}\right)$ one has

$$
\begin{aligned}
\beta\left(r_{1}, r_{2}\right) & \geq \frac{\int_{\Omega} F(x, \eta) d x-\int_{\Omega} F\left(x, \theta_{1}\right) d x+\frac{\mu}{\lambda}\left(G_{\eta}-G^{\theta_{1}}\right)}{\Phi(w)-\Phi(u)} \\
& \geq \frac{\int_{\Omega} F(x, \eta) d x-\int_{\Omega} F\left(x, \theta_{1}\right) d x+\frac{\mu}{\lambda}\left(G_{\eta}-G^{\theta_{1}}\right)}{\frac{\eta^{p^{+}}}{p^{-}}\|a\|_{1}} .
\end{aligned}
$$

Due to $\left(A_{2}\right)$ we get

$$
\alpha\left(r_{1}, r_{2}, r_{3}\right)<\beta\left(r_{1}, r_{2}\right) .
$$

Now, we show that the functional $I_{\lambda}$ satisfies the assumption $\left(a_{2}\right)$ of Theorem 1 . Let $u_{1}$ and $u_{2}$ be two local minima for $I_{\lambda}$. Then $u_{1}$ and $u_{2}$ are critical points for $I_{\lambda}$, and so, they are weak solutions for the problem $\left(P_{\lambda, \mu}^{f, g}\right)$. We want to prove that they are non-negative. Let $u_{0}$ be a (non-trivial) weak solution of the problem $\left(P_{\lambda, \mu}^{f, g}\right)$. Arguing by a contradiction, assume that the set $\mathcal{A}=\left\{x \in \Omega: u_{0}(x)<0\right\}$ is non-empty and of positive measure. Put $\bar{v}(x)=\min \left\{0, u_{0}(x)\right\}$ for all $x \in \Omega$. Clearly, $\bar{v} \in X$ and one has

$$
\begin{aligned}
& \int_{\Omega}\left(\left|\nabla u_{0}(x)\right|^{p(x)-2} \nabla u_{0}(x)+\frac{\left|\nabla u_{0}(x)\right|^{2 p(x)-2} \nabla u_{0}(x)}{\sqrt{1+\left|\nabla u_{0}(x)\right|^{2 p(x)}}}\right) \nabla \bar{v}(x) d x \\
+ & \int_{\Omega} a(x)\left|u_{0}(x)\right|^{p(x)-2} u_{0}(x) \bar{v}(x) d x \\
- & \lambda \int_{\Omega} f\left(x, u_{0}(x)\right) \bar{v}(x) d x-\mu \int_{\Omega} g\left(x, u_{0}(x)\right) \bar{v}(x) d x=0 .
\end{aligned}
$$

Since we could assume that $f$ is a non-negative, and $g$ is a non-negative, for fixed $\lambda>0$ and $\mu \geq 0$ and by choosing $\bar{v}(x)=u_{0}(x)$ one has

$$
\begin{aligned}
& \int_{\mathcal{A}}\left(\left|\nabla u_{0}(x)\right|^{p(x)}+\frac{\left|\nabla u_{0}(x)\right|^{2 p(x)}}{\sqrt{1+\left|\nabla u_{0}(x)\right|^{2 p(x)}}}\right) d x \\
+ & \int_{\mathcal{A}} a(x)\left|u_{0}(x)\right|^{p(x)} d x \\
= & \lambda \int_{\mathcal{A}} f\left(x, u_{0}(x)\right) u_{0}(x) d x+\mu \int_{\mathcal{A}} g\left(x, u_{0}(x)\right) u_{0}(x) d x \leq 0 .
\end{aligned}
$$

Hence, that is, $\left\|u_{0}\right\|_{w^{1, p(x)}(\mathcal{A})}=0$ which is an absurd. Hence, our claim is proved. Then, we observe $u_{1}(x) \geq 0$ and $u_{2}(x) \geq 0$ for every $x \in \Omega$. Thus, it follows that $(\lambda f+\mu g)\left(x, s u_{1}+(1-s) u_{2}\right) \geq 0$ for all $s \in[0,1]$, and consequently, $\Psi\left(s u_{1}+(1-\right.$ $\left.s) u_{2}\right) \geq 0$, for every $s \in[0,1]$. Hence, Theorem 1 implies that for every

$$
\lambda \in\left(\frac{\frac{\eta^{p^{+}}}{p^{-}}\|a\|_{1}}{\int_{\Omega} F(x, \eta) d x-\int_{\Omega} F\left(x, \theta_{1}\right) d x}\right.
$$


S. Heidarkhani, A.L.A. De Araujo, G.A. Afrouzi, S. Moradi - Multiple ...

$$
\left., \frac{1}{p^{+} k^{p^{-}}} \min \left\{\frac{\theta_{1}^{p^{-}}}{\int_{\Omega} F\left(x, \theta_{1}\right) d x}, \frac{\theta_{2}^{p^{-}}}{\int_{\Omega} F\left(x, \theta_{2}\right) d x}, \frac{\theta_{3}^{p^{-}}-\theta_{2}^{p^{-}}}{\int_{\Omega} F\left(x, \theta_{3}\right) d x}\right\}\right)
$$

and $\mu \in\left[0, \delta_{\lambda, g}\right)$, the functional $I_{\lambda}$ has three critical points $u_{i}, i=1,2,3$, in $X$ such that $\Phi\left(u_{1}\right)<r_{1}, \Phi\left(u_{2}\right)<r_{2}$ and $\Phi\left(u_{3}\right)<r_{2}+r_{3}$, that is,

$$
\max _{x \in \Omega}\left|u_{1}(x)\right|<\theta_{1}, \max _{x \in \Omega}\left|u_{2}(x)\right|<\theta_{2} \text { and } \max _{x \in \Omega}\left|u_{3}(x)\right|<\theta_{3} .
$$

Then, taking into account the fact that the weak solutions of the problem $\left(P_{\lambda, \mu}^{f, g}\right)$ are exactly critical points of the functional $I_{\lambda}$ we have the desired conclusion.

Remark 1. We observe that, in Theorem 3, no asymptotic conditions on $f$ and $g$ are needed and only algebraic conditions on $f$ are imposed to guarantee the existence of the weak solutions.

For positive constants $\theta_{1} \geq k, \theta_{4}$ and $\eta \geq 1$, set

$$
\begin{aligned}
& \delta_{\lambda, g}^{\prime}:=\min \left\{\frac { 1 } { k ^ { p ^ { - } } p ^ { + } } \operatorname { m i n } \left\{\frac{\theta_{1}^{p^{-}}-\lambda k^{p^{-}} p^{+} \int_{\Omega} F\left(x, \theta_{1}\right) d x}{G^{\theta_{1}}}\right.\right. \\
& \left., \frac{\theta_{4}^{p^{-}}-2 \lambda k^{p^{-}} p^{+} \int_{\Omega} F\left(x, \frac{1}{p^{-}} \theta_{4}\right) d x}{2 G^{\frac{1}{p^{-}} \theta_{4}}}, \frac{\theta_{4}^{p^{-}}-2 \lambda k^{p^{-}} p^{+} \int_{\Omega} F\left(x, \theta_{4}\right) d x}{2 G^{\theta_{4}}}\right\} \\
& \left., \frac{\frac{\eta^{p^{+}}}{p^{-}}\|a\|_{1}-\lambda\left(\int_{\Omega} F(x, \eta) d x-\int_{\Omega} F\left(x, \theta_{1}\right) d x\right)}{G_{\eta}-G^{\theta_{1}}}\right\} .
\end{aligned}
$$

Now, we deduce the following straightforward consequence of Theorem 3.

Theorem 4. Assume that there exist positive constants $\theta_{1} \geq k, \theta_{4}$ and $\eta \geq 1$ with $\theta_{1}<\min \left\{\eta^{\frac{p^{+}}{p^{-}}}, \sqrt[p]{\|a\|_{1}} k \eta\right\}$ and $\max \left\{\eta, \sqrt[p^{-}]{\frac{2 p^{+}\|a\|_{1}}{p^{-}}} k \eta^{\frac{p^{+}}{p^{-}}}\right\}<\theta_{4}$ such that

$\left(A_{3}\right) f(x, t) \geq 0$ for each $(x, t) \in \Omega \times\left[-\theta_{4}, \theta_{4}\right]$;

$$
\max \left\{\frac{\int_{\Omega} F\left(x, \theta_{1}\right) d x}{\theta_{1}^{p^{-}}}, \frac{2 \int_{\Omega} F\left(x, \theta_{4}\right) d x}{\theta_{4}^{p^{-}}}\right\}<\frac{p^{-}}{p^{-}+k^{p^{-}} p^{+}\|a\|_{1}} \frac{\int_{\Omega} F(x, \eta) d x}{\eta^{p^{+}}}
$$


S. Heidarkhani, A.L.A. De Araujo, G.A. Afrouzi, S. Moradi - Multiple ...

Then, for every

$$
\lambda \in\left(\frac{\frac{p^{-}+k^{p^{-}} p^{+}\|a\|_{1}}{p^{+} p^{-} k^{p^{-}}} \eta^{p^{+}}}{\int_{\Omega} F(x, \eta) d x}, \frac{1}{p^{+} k^{p^{-}}} \min \left\{\frac{\theta_{1}^{p^{-}}}{\int_{\Omega} F\left(x, \theta_{1}\right) d x}, \frac{\theta_{4}^{p^{-}}}{2 \int_{\Omega} F\left(x, \theta_{4}\right) d x}\right\}\right)
$$

and for every non-negative $L^{1}$-Carathéodory function $g: \Omega \times \mathbb{R} \rightarrow \mathbb{R}$, there exists $\delta_{\lambda, g}^{\prime}>0$ given by (6) such that, for each $\mu \in\left[0, \delta_{\lambda, g}^{\prime}\right)$, the problem $\left(P_{\lambda, \mu}^{f, g}\right)$ possesses at least three non-negative weak solutions $u_{1}, u_{2}$ and $u_{3}$ such that

$$
\max _{x \in \Omega}\left|u_{1}(x)\right|<\theta_{1}, \max _{x \in \Omega}\left|u_{2}(x)\right|<\frac{1}{\sqrt[p^{-}]{2}} \theta_{4} \text { and } \max _{x \in \Omega}\left|u_{3}(x)\right|<\theta_{4} .
$$

Proof. Choose $\theta_{2}=\frac{1}{p^{-} \sqrt{2}} \theta_{4}$ and $\theta_{3}=\theta_{4}$. So, from $\left(A_{4}\right)$ one has

$$
\begin{aligned}
\frac{\int_{\Omega} F\left(x, \theta_{2}\right) d x}{\theta_{2}^{p^{-}}} & =\frac{2 \int_{\Omega} F\left(x, \frac{1}{p^{-}} \theta_{4}\right) d x}{\theta_{4}^{p^{-}}} \leq \frac{2 \int_{\Omega} F\left(x, \theta_{4}\right) d x}{\theta_{4}^{p^{-}}} \\
& <\frac{p^{-}}{p^{-}+k^{p^{-}} p^{+}\|a\|_{1}} \frac{\int_{\Omega} F(x, \eta) d x}{\eta^{p^{+}}}
\end{aligned}
$$

and

$$
\frac{\int_{\Omega} F\left(x, \theta_{3}\right) d x}{\theta_{3}^{p^{-}}-\theta_{2}^{p^{-}}}=\frac{2 \int_{\Omega} F\left(x, \theta_{4}\right) d x}{\theta_{4}^{p^{-}}}<\frac{p^{-}}{p^{-}+k^{p^{-}} p^{+}\|a\|_{1}} \frac{\int_{\Omega} F(x, \eta) d x}{\eta^{p^{+}}}
$$

Moreover, taking into account that $\theta_{1}<\eta^{\frac{p^{+}}{p^{-}}}$, by using $\left(A_{4}\right)$ we have

$$
\begin{aligned}
& \frac{p^{-}}{k^{p^{-}} p^{+}\|a\|_{1}} \frac{\int_{\Omega} F(x, \eta) d x-\int_{\Omega} F\left(x, \theta_{1}\right) d x}{\eta^{p^{+}}} \\
> & \frac{p^{-}}{k^{p^{-}} p^{+}\|a\|_{1}} \frac{\int_{\Omega} F(x, \eta) d x}{\eta^{p^{+}}}-\frac{p^{-}}{k^{p^{-}} p^{+}\|a\|_{1}} \frac{\int_{\Omega} F\left(x, \theta_{1}\right) d x}{\theta_{1}^{p^{-}}} \\
> & \frac{p^{-}}{k^{p^{-}} p^{+}\|a\|_{1}}\left(\frac{\int_{\Omega} F(x, \eta) d x}{\eta^{p^{+}}}-\frac{p^{-}}{p^{-}+k^{p^{-}} p^{+}\|a\|_{1}} \frac{\int_{\Omega} F(x, \eta) d x}{\eta^{p^{+}}}\right) \\
= & \frac{p^{-}}{p^{-}+k^{p^{-}} p^{+}\|a\|_{1}} \frac{\int_{\Omega} F(x, \eta) d x}{\eta^{p^{+}}} .
\end{aligned}
$$

Hence, from $\left(A_{4}\right),(7)$ and $(8)$, it is easy to see that the assumption $\left(A_{2}\right)$ of Theorem 3 is satisfied, and it follows the conclusion.

We now present the following example to illustrate Theorem 4. 
S. Heidarkhani, A.L.A. De Araujo, G.A. Afrouzi, S. Moradi - Multiple ...

Example 1. Let $\Omega=\left\{(x, y) \in \mathbb{R}^{2}: x^{2}+y^{2}<10^{-4}\right\}$. We consider the problem

$$
\begin{cases}-\operatorname{div}\left(\left(1+\frac{|\nabla u|^{p(x, y)}}{\sqrt{1+|\nabla u|^{2 p(x, y)}}}\right)|\nabla u|^{p(x, y)-2} \nabla u\right)+a(x, y)|u|^{p(x, y)-2} u & \\ =\lambda f(x, y, u)+\mu g(x, y, u), & \text { in } \Omega, \\ \frac{\partial u}{\partial \nu}=0, & \text { on } \partial \Omega\end{cases}
$$

where $p(x, y)=x^{2}+y^{2}+4$ for every $x, y \in \Omega, a(x, y)=4\left(x^{2}+y^{2}\right)+2$ for every $x, y \in \Omega$ and

$$
f(x, y, t)= \begin{cases}104\left(x^{2}+y^{2}\right) t^{12}, & \text { if } t \leq 1 \\ 8\left(x^{2}+y^{2}\right)\left(13 t-\frac{3 \pi}{2} \sin \left(\frac{3 \pi}{2} t\right)\right), & \text { if } t>1\end{cases}
$$

By the expression of $f$, we have

$$
F(x, y, t)= \begin{cases}8\left(x^{2}+y^{2}\right) t^{13}, & \text { if } t \leq 1 \\ 8\left(x^{2}+y^{2}\right)\left(\frac{13}{2} t^{2}+\cos \left(\frac{3 \pi}{2} t\right)-\frac{11}{2}\right), & \text { if } t>1\end{cases}
$$

By simple calculations, we obtain $k=\sqrt[4]{\frac{4}{\pi}} \frac{\left(1+10^{-4} \pi\right)}{\left(10^{-8}+10^{-4}\right)^{\frac{1}{4}}}, p^{-}=4$ and $p^{+}=4+10^{-4}$. Taking $\theta_{1}=\frac{3}{10}, \theta_{4}=10^{8}$ and $\eta=1$, then all conditions in Theorem 4 are satisfied. Therefore, it follows that for each

$$
\lambda \in\left(\frac{4+2 k^{4} \pi\left(4+10^{-4}\right)\left(10^{-8}+10^{-4}\right)}{16 k^{4} \pi\left(4+10^{-4}\right) \times 10^{-8}}, \frac{10^{9}}{4 \times 3^{9} k^{4} \pi\left(4+10^{-4}\right) \times 10^{-8}}\right)
$$

there exists $\delta>0$ and for every non-negative $L^{1}$-Carathéodory function $g: \Omega \times \mathbb{R} \rightarrow$ $\mathbb{R}$, there exists $\delta>0$ such that, for each $\mu \in[0, \delta)$, the problem (9) possesses at least three non-negative weak solutions $u_{1}, u_{2}$ and $u_{3}$ such that

$$
\max _{x \in \Omega}\left|u_{1}(x)\right|<\frac{3}{10}, \max _{x \in \Omega}\left|u_{2}(x)\right|<\frac{1}{\sqrt[4]{2}} 10^{8} \text { and } \max _{x \in \Omega}\left|u_{3}(x)\right|<10^{8} .
$$

We want to point out a simple consequence of Theorem 4, in which the function $f$ has separated variables.

Theorem 5. Let $f_{1} \in L^{1}(\Omega)$ and $f_{2} \in C(\mathbb{R})$ be two functions. Put $\tilde{F}(t)=\int_{0}^{t} f_{2}(\xi) d \xi$ for all $t \in \mathbb{R}$ and assume that there exist positive constants $\theta_{1} \geq k, \theta_{4}$ and $\eta \geq 1$ with $\theta_{1}<\min \left\{\eta^{\frac{p^{+}}{p^{-}}}, \sqrt[p^{-}]{\|a\|_{1}} k \eta\right\}$ and $\max \left\{\eta, \sqrt[p^{-}]{\frac{2 p^{+}\|a\|_{1}}{p^{-}}} k \eta^{\frac{p^{+}}{p^{-}}}\right\}<\theta_{4}$ such that

$\left(A_{5}\right) f_{1}(x) \geq 0$ for each $x \in \Omega$ and $f_{2}(t) \geq 0$ for each $t \in\left[-\theta_{4}, \theta_{4}\right]$;

$\left(A_{6}\right) \quad \max \left\{\frac{\sup _{|t| \leq \theta_{1}} \tilde{F}(t)}{\theta_{1}^{p^{-}}}, \frac{2 \sup _{|t| \leq \theta_{4}} \tilde{F}(t)}{\theta_{4}^{p^{-}}}\right\}<\frac{p^{-}}{p^{-}+k^{p^{-}} p^{+}\|a\|_{1}} \frac{\tilde{F}(\eta)}{\eta^{p^{+}}}$. 
S. Heidarkhani, A.L.A. De Araujo, G.A. Afrouzi, S. Moradi - Multiple ...

Then, for every

$$
\lambda \in\left(\frac{\frac{p^{-}+k^{p^{-}} p^{+}\|a\|_{1}}{p^{+} p^{-} k^{p^{-}}} \eta^{p^{+}}}{\tilde{F}(\eta) \int_{\Omega} f_{1}(x) d x}, \frac{1}{p^{+} k^{p^{-}} \int_{\Omega} f_{1}(x) d x} \min \left\{\frac{\theta_{1}^{p^{-}}}{\sup _{|t| \leq \theta_{1}} \tilde{F}(t)}, \frac{\theta_{4}^{p^{-}}}{2 \sup _{|t| \leq \theta_{4}} \tilde{F}(t)}\right\}\right)
$$

and for every non-negative $L^{1}$-Carathéodory function $g: \Omega \times \mathbb{R} \rightarrow \mathbb{R}$, for each

$$
\begin{aligned}
& \mu \in\left[0, \min \left\{\frac { 1 } { k ^ { p ^ { - } } p ^ { + } } \operatorname { m i n } \left\{\frac{\theta_{1}^{p^{-}}-\lambda k^{p^{-}} p^{+} \sup _{|t| \leq \theta_{1}} \tilde{F}(t) \int_{\Omega} f_{1}(x) d x}{G^{\theta_{1}}}\right.\right.\right. \\
& , \frac{\theta_{4}^{p^{-}}-2 \lambda k^{p^{-}} p^{+} \sup _{|t| \leq \frac{1}{p^{-} \sqrt{2}} \theta_{4}} \tilde{F}(t) \int_{\Omega} f_{1}(x) d x}{2 G^{\frac{1}{p^{-} \sqrt{2}} \theta_{4}}} \\
& \left., \frac{\theta_{4}^{p^{-}}-2 \lambda k^{p^{-}} p^{+} \sup _{|t| \leq \theta_{4}} \tilde{F}(t) \int_{\Omega} f_{1}(x) d x}{2 G^{\theta_{4}}}\right\} \\
& \left.\left., \frac{\frac{\eta^{p^{+}}}{p^{-}}\|a\|_{1}-\lambda \int_{\Omega} f_{1}(x) d x\left(\tilde{F}(\eta)-\sup _{|t| \leq \theta_{1}} \tilde{F}(t)\right)}{G_{\eta}-G^{\theta_{1}}}\right\}\right),
\end{aligned}
$$

the problem

$$
\begin{cases}-\operatorname{div}\left(\left(1+\frac{|\nabla u|^{p(x)}}{\sqrt{1+|\nabla u|^{2 p(x)}}}\right)|\nabla u|^{p(x)-2} \nabla u\right)+a(x)|u|^{p(x)-2} u & \\ =\lambda f_{1}(x) f_{2}(u)+\mu g(x, u), & \text { in } \Omega, \\ \frac{\partial u}{\partial \nu}=0, & \text { on } \partial \Omega\end{cases}
$$

possesses at least three non-negative weak solutions $u_{1}, u_{2}$ and $u_{3}$ such that

$$
\max _{x \in \Omega}\left|u_{1}(x)\right|<\theta_{1}, \max _{x \in \Omega}\left|u_{2}(x)\right|<\frac{1}{\sqrt[p^{-}]{2}} \theta_{4} \text { and } \max _{x \in \Omega}\left|u_{3}(x)\right|<\theta_{4} .
$$

Proof. Set $f(x, u)=f_{1}(x) f_{2}(u)$ for each $(x, u) \in \Omega \times \mathbb{R}$. Since

$$
F(x, t)=f_{1}(x) \tilde{F}(t)
$$

from $\left(A_{5}\right)$ and $\left(A_{6}\right)$ we obtain $\left(A_{3}\right)$ and $\left(A_{4}\right)$, respectively.

Here, we present a simple consequence of Theorem 4 in the case $f$ does not depend upon $x$. 
S. Heidarkhani, A.L.A. De Araujo, G.A. Afrouzi, S. Moradi - Multiple ...

Theorem 6. Assume that there exist positive constants $\theta_{1} \geq k, \theta_{4}$ and $\eta \geq 1$ with $\theta_{1}<\min \left\{\eta^{\frac{p^{+}}{p^{-}}}, \sqrt[p]{\|a\|_{1}} k \eta\right\}$ and $\max \left\{\eta, \sqrt[p^{-}]{\frac{2 p^{+}\|a\|_{1}}{p^{-}}} k \eta^{\frac{p^{+}}{p^{-}}}\right\}<\theta_{4}$ such that

$\left(A_{7}\right) f(t) \geq 0$ for each $t \in\left[-\theta_{4}, \theta_{4}\right]$;

$$
\max \left\{\frac{F\left(\theta_{1}\right)}{\theta_{1}^{p^{-}}}, \frac{2 F\left(\theta_{4}\right)}{\theta_{4}^{p^{-}}}\right\}<\frac{p^{-}}{p^{-}+k^{p^{-}} p^{+}\|a\|_{1}} \frac{F(\eta)}{\eta^{p^{+}}} .
$$

Then, for every

$$
\lambda \in\left(\frac{\frac{p^{-}+k^{p^{-}} p^{+}\|a\|_{1}}{p^{+} p^{-} k^{p^{-}}} \eta^{p^{+}}}{\operatorname{meas}(\Omega) F(\eta)}, \frac{1}{p^{+} k^{p^{-}} \operatorname{meas}(\Omega)} \min \left\{\frac{\theta_{1}^{p^{-}}}{F\left(\theta_{1}\right)}, \frac{\theta_{4}^{p^{-}}}{2 F\left(\theta_{4}\right)}\right\}\right)
$$

and for every non-negative $L^{1}$-Carathéodory function $g: \Omega \times \mathbb{R} \rightarrow \mathbb{R}$, for each

$$
\begin{aligned}
& \mu \in\left[0, \min \left\{\frac { 1 } { k ^ { p ^ { - } } p ^ { + } } \operatorname { m i n } \left\{\frac{\theta_{1}^{p^{-}}-\lambda k^{p^{-}} p^{+} \operatorname{meas}(\Omega) F\left(\theta_{1}\right)}{G^{\theta_{1}}}\right.\right.\right. \\
& \left., \frac{\theta_{4}^{p^{-}}-2 \lambda k^{p^{-}} p^{+} \operatorname{meas}(\Omega) F\left(\frac{1}{p^{-} \sqrt{2}} \theta_{4}\right)}{2 G^{\frac{1}{p^{-}} \theta_{4}}}, \frac{\theta_{4}^{p^{-}}-2 \lambda k^{p^{-}} p^{+} \operatorname{meas}(\Omega) F\left(\theta_{4}\right)}{2 G^{\theta_{4}}}\right\} \\
& \left.\left., \frac{\frac{\eta^{p^{+}}}{p^{-}}\|a\|_{1}-\lambda \operatorname{meas}(\Omega)\left(F(\eta)-F\left(\theta_{1}\right)\right)}{G_{\eta}-G^{\theta_{1}}}\right\}\right),
\end{aligned}
$$

the problem

$$
\begin{cases}-\operatorname{div}\left(\left(1+\frac{|\nabla u|^{p(x)}}{\sqrt{1+|\nabla u|^{2 p(x)}}}\right)|\nabla u|^{p(x)-2} \nabla u\right)+a(x)|u|^{p(x)-2} u & \\ =\lambda f(u)+\mu g(x, u), & \text { in } \Omega, \\ \frac{\partial u}{\partial \nu}=0, & \text { on } \partial \Omega\end{cases}
$$

possesses at least three non-negative weak solutions $u_{1}, u_{2}$ and $u_{3}$ such that

$$
\max _{x \in \Omega}\left|u_{1}(x)\right|<\theta_{1}, \max _{x \in \Omega}\left|u_{2}(x)\right|<\frac{1}{\sqrt[p]{2}} \theta_{4} \text { and } \max _{x \in \Omega}\left|u_{3}(x)\right|<\theta_{4} .
$$

The following result is a consequence of Theorem 4 when $\mu=0$.

Theorem 7. Let $f: \Omega \times \mathbb{R} \rightarrow \mathbb{R}$ be a continuous function such that $t f(x, t)>0$ for all $(x, t) \in \Omega \times(\mathbb{R} \backslash\{0\})$. Assume that 
$\left(A_{9}\right) \lim _{t \rightarrow 0} \frac{f(x, t)}{|t|^{p^{-}-1}}=\lim _{|t| \rightarrow \infty} \frac{f(x, t)}{|t|^{p^{-}-1}}=0$.

Then, for every $\lambda>\bar{\lambda}$ where

$$
\begin{gathered}
\bar{\lambda}=\frac{p^{-}+k^{p^{-}} p^{+}\|a\|_{1}}{p^{+} p^{-} k^{p^{-}}} \max \left\{\inf _{\eta \geq 1} \frac{\eta^{p^{+}}}{\int_{\Omega} F(x, \eta) d x} ; \inf _{0<\eta<1} \frac{\eta^{p^{-}}}{\int_{\Omega} F(x, \eta) d x}\right. \\
\left.\inf _{-1<\eta<0} \frac{(-\eta)^{p^{-}}}{\int_{\Omega} F(x, \eta) d x} ; \inf _{\eta \leq-1} \frac{(-\eta)^{p^{+}}}{\int_{\Omega} F(x, \eta) d x}\right\},
\end{gathered}
$$

the problem $\left(P_{\lambda, \mu}^{f, g}\right)$, in the case $\mu=0$ possesses at least four distinct non-trivial solutions.

Proof. Set

$$
f_{1}(x, t)= \begin{cases}f(x, t), & \text { if }(x, t) \in \Omega \times[0,+\infty), \\ 0, & \text { otherwise }\end{cases}
$$

and

$$
f_{2}(x, t)= \begin{cases}-f(x,-t), & \text { if }(x, t) \in \Omega \times[0,+\infty), \\ 0, & \text { otherwise }\end{cases}
$$

and define $F_{1}(x, t):=\int_{0}^{t} f_{1}(x, \xi) d \xi$ for every $(x, t) \in \Omega \times \mathbb{R}$. Fix $\lambda>\lambda^{*}$, and let $\eta \geq 1$ such that $\lambda>\frac{\left(\frac{p^{-}+k^{p^{-}} p^{+}\|a\|_{1}}{p^{+} p^{-} p^{-}}\right) \eta^{p^{+}}}{\int_{\Omega} F_{1}(x, \eta) d x}$. From

$$
\lim _{t \rightarrow 0^{+}} \frac{f_{1}(x, t)}{t^{p^{-}-1}}=\lim _{t \rightarrow+\infty} \frac{f_{1}(x, t)}{t^{p^{-}-1}}=0
$$

there is $\theta_{1} \geq k$ such that

$$
\theta_{1}<\min \left\{\eta^{\frac{p^{+}}{p^{-}}}, \sqrt[p^{-}]{\|a\|_{1}} k \eta\right\} \text { and } \frac{\int_{\Omega} F_{1}\left(x, \theta_{1}\right) d x}{\theta_{1}^{p^{-}}}<\frac{1}{\lambda k^{p^{-}} p^{+}}
$$

and there is $\theta_{4}>0$ such that

$$
\max \left\{\eta, \sqrt[p^{-}]{\frac{2 p^{+}\|a\|_{1}}{p^{-}}} k \eta^{\frac{p^{+}}{p^{-}}}\right\}<\theta_{4} \text { and } \frac{\int_{\Omega} F_{1}\left(x, \theta_{4}\right) d x}{\theta_{4}^{p^{-}}}<\frac{1}{2 \lambda k^{p^{-} p^{+}}} .
$$

Then, $\left(A_{4}\right)$ in Theorem 4 is satisfied,

$$
\lambda \in\left(\frac{\frac{p^{-}+k^{p^{-}} p^{+}\|a\|_{1}}{p^{+} p^{-} k^{p^{-}}} \eta^{p^{+}}}{\int_{\Omega} F_{1}(x, \eta) d x}, \frac{1}{p^{+} k^{p^{-}}} \min \left\{\frac{\theta_{1}^{p^{-}}}{\int_{\Omega} F_{1}\left(x, \theta_{1}\right) d x}, \frac{\theta_{4}^{p^{-}}}{2 \int_{\Omega} F_{1}\left(x, \theta_{4}\right) d x}\right\}\right) .
$$


S. Heidarkhani, A.L.A. De Araujo, G.A. Afrouzi, S. Moradi - Multiple ...

Hence, the problem $\left(P_{\lambda, \mu}^{f_{1}, g}\right)$, in the case $\mu=0$ admits two positive solutions $u_{1}, u_{2}$, which are positive solutions of the problem $\left(P_{\lambda, \mu}^{f, g}\right)$, in the case $\mu=0$. Next, arguing in the same way, from

$$
\lim _{t \rightarrow 0^{+}} \frac{f_{2}(x, t)}{t^{p^{-}-1}}=\lim _{t \rightarrow+\infty} \frac{f_{2}(x, t)}{t^{p^{-}-1}}=0
$$

we ensure the existence of two positive solutions $u_{3}, u_{4}$ for the problem $\left(P_{\lambda, \mu}^{f_{2}, g}\right)$, in the case $\mu=0$. Clearly, $-u_{3},-u_{4}$ are negative solutions of the problem $\left(P_{\lambda, \mu}^{f, g}\right)$, in the case $\mu=0$ and the conclusion is achieved.

Theorem 8. We explicitly observe that in Theorem 7 no symmetric condition on $f$ is assumed. However, whenever $f$ is an odd continuous non-zero function such that $f(x, t) \geq 0$ for all $(x, t) \in \Omega \times[0,+\infty),\left(A_{9}\right)$ can be replaced by

$\left(A_{10}\right) \lim _{t \rightarrow 0^{+}} \frac{f(x, t)}{t^{p^{-}-1}}=\lim _{t \rightarrow \infty} \frac{f(x, t)}{t^{p^{-}-1}}=0$,

ensuring the existence of at least four distinct non-trivial solutions the problem $\left(P_{\lambda, \mu}^{f, g}\right)$, in the case $\mu=0$ for every $\lambda>\lambda^{*}$ where

$$
\lambda^{*}=\inf _{\eta \geq 1} \frac{\frac{p^{-}+k^{p^{-}} p^{+}\|a\|_{1}}{p^{+} p^{-} k^{p^{-}}} \eta^{p^{+}}}{\int_{\Omega} F(x, \eta) d x} .
$$

We end this paper by presenting the following version of Theorem 3, in the case $p(x)=p$ for every $x \in \Omega$.

Theorem 9. Let $p(x)=p>N$ for every $x \in \Omega$. Assume that there exist positive constants $\theta_{1} \geq k, \theta_{2}, \theta_{3}$ and $\eta \geq 1$ with $\theta_{1}<\sqrt[p]{\|a\|_{1}} k \eta$, $\max \left\{\eta, \sqrt[p]{\|a\|_{1}} k \eta\right\}<\theta_{2}$ and $\theta_{2}<\theta_{3}$ such that

$\left(A_{11}\right) f(x, t) \geq 0$ for each $(x, t) \in \Omega \times\left[-\theta_{3}, \theta_{3}\right]$;

$$
\begin{aligned}
& \max \left\{\frac{\int_{\Omega} F\left(x, \theta_{1}\right) d x}{\theta_{1}^{p}}, \frac{\int_{\Omega} F\left(x, \theta_{2}\right) d x}{\theta_{2}^{p}}, \frac{\int_{\Omega} F\left(x, \theta_{3}\right) d x}{\theta_{3}^{p}-\theta_{2}^{p}}\right\} \\
& <\frac{1}{k^{p}\|a\|_{1}} \frac{\int_{\Omega} F(x, \eta) d x-\int_{\Omega} F\left(x, \theta_{1}\right) d x}{\eta^{p}} .
\end{aligned}
$$

Then, for every

$$
\lambda \in\left(\frac{\frac{\eta^{p}}{p}\|a\|_{1}}{\int_{\Omega} F(x, \eta) d x-\int_{\Omega} F\left(x, \theta_{1}\right) d x}\right.
$$


S. Heidarkhani, A.L.A. De Araujo, G.A. Afrouzi, S. Moradi - Multiple ...

$$
\left., \frac{1}{p k^{p}} \min \left\{\frac{\theta_{1}^{p}}{\int_{\Omega} F\left(x, \theta_{1}\right) d x}, \frac{\theta_{2}^{p}}{\int_{\Omega} F\left(x, \theta_{2}\right) d x}, \frac{\theta_{3}^{p}-\theta_{2}^{p}}{\int_{\Omega} F\left(x, \theta_{3}\right) d x}\right\}\right)
$$

for every non-negative $L^{1}$-Carathéodory function $g: \Omega \times \mathbb{R} \rightarrow \mathbb{R}$ for each $\mu \in\left[0, \delta_{\lambda, g}\right)$,

$$
\begin{gathered}
\mu \in\left[0, \min \left\{\frac { 1 } { k ^ { p } p } \operatorname { m i n } \left\{\frac{\theta_{1}^{p}-\lambda k^{p} p \int_{\Omega} F\left(x, \theta_{1}\right) d x}{G^{\theta_{1}}}\right.\right.\right. \\
\left., \frac{\theta_{2}^{p}-\lambda k^{p} p \int_{\Omega} F\left(x, \theta_{2}\right) d x}{G^{\theta_{2}}}, \frac{\left(\theta_{3}^{p}-\theta_{2}^{p}\right)-\lambda k^{p} p \int_{\Omega} F\left(x, \theta_{3}\right) d x}{G^{\theta_{3}}}\right\} \\
\left.\left., \frac{\frac{\eta^{p}}{p}\|a\|_{1}-\lambda\left(\int_{\Omega} F(x, \eta) d x-\int_{\Omega} F\left(x, \theta_{1}\right) d x\right)}{G_{\eta}-G^{\theta_{1}}}\right\}\right) .
\end{gathered}
$$

the problem

$$
\begin{cases}-\operatorname{div}\left(\left(1+\frac{|\nabla u|^{p}}{\sqrt{1+|\nabla u|^{2 p}}}\right)|\nabla u|^{p-2} \nabla u\right)+a(x)|u|^{p-2} u & \\ =\lambda f(x, u(x))+\mu g(x, u), & \text { in } \Omega, \\ \frac{\partial u}{\partial \nu}=0, & \text { on } \partial \Omega\end{cases}
$$

possesses at least three non-negative weak solutions $u_{1}, u_{2}$, and $u_{3}$ such that

$$
\max _{x \in \Omega}\left|u_{1}(x)\right|<\theta_{1}, \max _{x \in \Omega}\left|u_{2}(x)\right|<\theta_{2} \text { and } \max _{x \in \Omega}\left|u_{3}(x)\right|<\theta_{3} .
$$

\section{REFERENCES}

[1] G.A. Afrouzi, G. Caristi, D. Barilla, S. Moradi, A variational approach to perturbed three-point boundary value problems of Kirchhoff-type, Complex Var. Elliptic Eqs. 62 (2017), 397-412.

[2] S. Antontsev, S. Shmarev, Handbook of Differential Equations, Stationary Partial Differential Equations, vol. 3, (2006) (Chapter 1).

[3] M. Avci, Ni-Serrin type equations arising from capillarity phenomena with nonstandard growth, Bound. Value Probl. 2013 (2013):55.

[4] C. Bereanu, J. Mawhin, Boundary value problems with non-surjective $\phi$ Laplacian and one-sided bounded nonlinearity, Adv. Differ. Equ. 11 (2006), 35-60.

[5] G. Bin, On superlinear $p(x)$-Laplacian-like problem without Ambrosetti and Rabinowitz condition, Bull. Korean Math. Soc. 51 (2014), 409-421. 
S. Heidarkhani, A.L.A. De Araujo, G.A. Afrouzi, S. Moradi - Multiple ...

[6] G. Bonanno, P. Candito, Non-differentiable functionals and applications to elliptic problems with discontinuous nonlinearities, J. Differ. Equ. 244 (2008), 30313059.

[7] G. Bonanno, B. Di Bella, A boundary value problem for fourth-order elastic beam equations, J. Math. Anal. Appl. 343 (2008), 1166-1176.

[8] G. Bonanno, R. Livrea, J. Mawhin, Existence results for parametric boundary value problems involving the mean curvature operator, Nonlinear Differ. Equ. Appl. 22 (2015), 411-426.

[9] D. Bonheure, P. Habets, F. Obersnel, P. Omari, Classical and non-classical positive solutions of a prescribed curvature equation with singularities, Rend. Istit. Mat. Univ. Trieste 39 (2007), 63-85.

[10] E. Cabanillas Lapa, V. Pardo Rivera, J. Quique Broncano, No-flux boundary problems involving $p(x)$-Laplacian-like operators, Electron. J. Diff. Equ., Vol. 2015 (2015), No. 219, pp. 1-10.

[11] F. Cammaroto, L. Vilasi, Multiplicity results for a Neumann boundary value problem involving the $p(x)$-laplacian, Taiwanese J. Math. 16 (2012), 621-634.

[12] K.C. Chang, Critical Point Theory and Applications, Shanghai Scientific and Technology Press, Shanghai, (1986).

[13] Y. Chen, S. Levine, R. Rao, Variable exponent, linear growth functionals in image restoration, SIAM J. Appl. Math. 66 (2006), 1383-1406.

[14] P. Concus, P. Finn, A singular solution of the capillary equation I, II, Invent. Math. 29 (1975) (143-148), 149-159.

[15] G. D'Aguì, A. Sciammetta, Infinitely many solutions to elliptic problems with variable exponent and nonhomogeneous Neumann conditions, Nonlinear Anal. TMA 75 (2012),5612-5619.

[16] A.L.A. De Araujo, M. Montenegro, The mean curvature equation with oscillating nonlinearity, Adv. Nonlinear Stud. 15 (2015), 183-189.

[17] S.G. Deng, A local mountain pass theorem and applications to a double perturbed p(x)-Laplacian equations, Appl. Math. Comput. 211 (2009), 234-241.

[18] X.L. Fan, S.G. Deng, Multiplicity of positive solutions for a class of inhomogeneous Neumann problems involving the $p(x)$-Laplacian, Nonlinear Differ. Equ. Appl. NoDEA 16 (2009), 255-271.

[19] X.L. Fan, Q.H. Zhang, Existence of solutions for $p(x)$-Laplacian Dirichlet problem, Nonlinear Anal. TMA 52 (2003), 1843-1852.

[20] X.L. Fan, D. Zhao, On the generalize Orlicz-Sobolev space $W^{k, p(x)}(\Omega)$, J. Gansu Educ. College 12 (1998), 1-6. 
S. Heidarkhani, A.L.A. De Araujo, G.A. Afrouzi, S. Moradi - Multiple ...

[21] X.L Fan, D. Zhao, On the spaces $L^{p(x)}(\Omega)$ and $W^{m, p(x)}(\Omega)$, J. Math. Anal. Appl. 263 (2001), 424-446.

[22] F. Faraci, A note on the existence of infinitely many solutions for the one dimensional prescribed curvature equation, Stud. Univ. Babeş-Bolyai Math. 55 (2010), 83-90.

[23] T.C. Halsey, Electrorheological fluids, Science 258 (1992), 761-766.

[24] P. Habets, P. Omari, Multiple positive solutions of a one-dimensional prescribed mean curvature problem, Commun. Contemp. Math. 9 (2007), 701-730.

[25] S. Heidarkhani, G.A. Afrouzi, S. Moradi, Variational approaches to $p(x)$-laplacian-like problems with Neumann condition originated from a capillary phenomena, International J. Nonlinear Sci. Numerical Simul., DOI: https://doi.org/10.1515/ijnsns-2017-0114.

[26] S. Heidarkhani, A.L.A. De Araujo, G.A. Afrouzi, S. Moradi, Multiple solutions for Kirchhoff-type problems with with variable exponent and nonhomogeneous Neumann conditions, Math. Nach. 291 (2018), 326-342.

[27] S. Heidarkhani, A. Salari, $p(x)$-Laplacian-like problems with Neumann condition originated from a capillary phenomena, J. Nonlinear Funct. Anal. 2018 (2018), Article ID 11, 1-23.

[28] O. Kováčik, J. Rákosník, On the spaces $L^{p(x)}(\Omega)$ and $W^{1, p(x)}(\Omega)$, Czechoslovak Math. 41 (1991), 592-618.

[29] M. Manuela Rodrigues, Multiplicity of solutions on a nonlinear eigenvalue problem for $p(x)$-Laplacian-like operators, Mediterr. J. Math. 9 (2012), 211-223.

[30] W.M. Ni, J. Serrin, Existence and non-existence theorems for ground states of quasilinear partial differential equations, The anomalous case, Atti Accd. Naz. Lincei. 77 (1986), 231-257.

[31] W.M. Ni, J. Serrin, Non-existence theorems for quasilinear partial differential equations, Rend. Circ. Mat. Palermo(2) Suppl. 8 (1985), 171-185.

[32] W.M. Ni, J. Serrin, Non-existence theorems for singular solutions of quasilinear partial differential equations, Comm. Pure Appl. Math. 39 (1986), 379-399.

[33] F. Obersnel, P. Omari, Positive solutions of the Dirichlet problem for the prescribed mean curvature equation, J. Differ. Equ. 249 (2010), 1674-1725.

[34] S. Ouaro, A. Ouedraogo, S. Soma, Multivalued problem with Robin boundary condition involving diffuse measure data and variable exponent, Adv. Nonlinear Anal. 3 (2014), 209-235.

[35] H. Pan, One-dimensional prescribed mean curvature equation with exponential nonlinearity, Nonlinear Anal. TMA 70 (2009), 999-1010. 
S. Heidarkhani, A.L.A. De Araujo, G.A. Afrouzi, S. Moradi - Multiple ...

[36] L.A. Peletier, J. Serrin, Ground states for the prescribed mean curvature equation, Proc. Amer. Math. Soc. 100 (1987), 694-700.

[37] C. Pfeiffer, C. Mavroidis, Y. Bar-Cohen, B. Dolgin, Electrorheological fluid based force feedback device, in Proceedings of the 1999 SPIE Telemanipulator and Telepresence Technologies VI Conference (Boston, MA), 3840 (1999), 88-99.

[38] V. Rădulescu, Nonlinear elliptic equations with variable exponent: old and new, Nonlinear Anal. TMA 121 (2015), 336-369.

[39] V. Rădulescu, D. Repovš, Partial Differential Equations with Variable Exponents, Variational Methods and Qualitative Analysis, Monographs and Research Notes in Mathematics, CRC Press, Boca Raton, FL, (2015).

[40] D. Repovš, Stationary waves of Schrödinger-type equations with variable exponent, Anal. Appl. 13 (2015), 645-661.

[41] M. Ružička, Electro-rheological Fluids: Modeling and Mathematical Theory Lecture Notes in Math., 1784, Springer, Berlin, (2000).

[42] S.G. Sanko, Denseness of $C_{0}^{\infty}\left(\mathbb{R}^{N}\right)$ in the generalized Sobolev spaces $W^{m, p(x)}\left(\mathbb{R}^{N}\right)$, Dokl. Ross. Akad. Nauk. 369 (1999), 451-454.

[43] M. Willem, Minimax Theorems, Birkhauser, Basel, (1996).

[44] E. Zeidler, Nonlinear Functional Analysis and its Applications, Vol. III, Springer, New York, (1985).

[45] V.V. Zhikov, Averaging of functionals in the calculus of variations and elasticity, Math. USSR Izv. 29 (1987), 33-66.

[46] Q.M. Zhou, On the superlinear problems involving $p(x)$-Laplacian-like operators without AR-condition, Nonlinear Anal. RWA 21 (2015), 161-169.

Shapour Heidarkhani

Department of Mathematics, Faculty of Sciences,

Razi University,

Kermanshah, Iran

email:s.heidarkhani@razi.ac.ir

Anderson L. A. De Araujo

Departamento de Matemática,

Universidade Federal de Viçosa, 36570-000,

Viçosa (MG), Brazil

email: anderson.araujo@ufv.br

Ghasem A. Afrouzi

Department of Mathematics, 
S. Heidarkhani, A.L.A. De Araujo, G.A. Afrouzi, S. Moradi - Multiple ...

Faculty of Mathematical Sciences, University of Mazandaran,

Babolsar, Iran

email:afrouzi@umz.ac.ir

Shahin Moradi

Department of Mathematics,

Faculty of Mathematical Sciences,

University of Mazandaran,

Babolsar, Iran

email: shahin.moradi86@yahoo.com 